\title{
Mannitol dosing error during interfacility transfer for intracranial emergencies
}

\author{
Cameron A. Elliott, MD, ${ }^{1}$ Mark MacKenzie, MD, ${ }^{2}$ and Cian J. O’Kelly, MD, MSc ${ }^{1}$ \\ ${ }^{1}$ Division of Neurosurgery, University of Alberta, and ²Department of Emergency Medicine, Royal Alexandra Hospital, Edmonton, \\ Alberta, Canada
}

\begin{abstract}
OBJECT Mannitol is commonly used to treat elevated intracranial pressure (ICP). The authors analyzed mannitol dosing errors at peripheral hospitals prior to or during transport to tertiary care facilities for intracranial emergencies. They also investigated the appropriateness of mannitol use based on the 2007 Brain Trauma Foundation guidelines for severe traumatic brain injury.
\end{abstract}

METHODS The authors conducted a retrospective review of the Shock Trauma Air Rescue Society (STARS) electronic patient database of helicopter medical evacuations in Alberta, Canada, between 2004 and 2012, limited to patients receiving mannitol before transfer. They extracted data on mannitol administration and patient characteristics, including diagnosis, mechanism, Glasgow Coma Scale score, weight, age, and pupil status.

RESULTS A total of 120 patients with an intracranial emergency received a mannitol infusion initiated at a peripheral hospital (median Glasgow Coma Scale score 6; range 3-13). Overall, there was a 22\% dosing error rate, which comprised an underdosing rate $(<0.25 \mathrm{~g} / \mathrm{kg}$ ) of $8.3 \%$ (10 of 120 patients), an overdosing rate $(>1.5 \mathrm{~g} / \mathrm{kg})$ of $7.5 \%(9$ of 120$)$, and a nonbolus administration rate (> 1 hour) of $6.7 \%$ (8 of 120). Overall, $72 \%$ of patients had a clear indication to receive mannitol as defined by meeting at least one of the following criteria based on Brain Trauma Foundation guidelines: neurological deterioration (11\%), severe traumatic brain injury (69\%), or pupillary abnormality $(25 \%)$.

CONCLUSIONS Mannitol administration at peripheral hospitals is prone to dosing error. Strategies such as a pretransport checklist may mitigate this risk.

http://thejns.org/doi/abs/10.3171/2014.11.JNS141596

KEY WORDS mannitol; dosing error; medical evacuation; trauma

$\mathrm{T}$ HE hyperosmotic agent mannitol is commonly used to treat raised intracranial pressure (ICP). In the acute presentation of an intracranial emergency, mannitol serves to bridge the gap between recognition and definitive treatment. This can be particularly important in patients requiring transportation from a peripheral hospital to a neurosurgical center, especially if transport is prolonged. The Brain Trauma Foundation (BTF) guidelines for in-hospital management of severe traumatic brain injury provide recommendations for mannitol use for the management of suspected or proven elevation in ICP. ${ }^{1}$ These widely used guidelines suggest that 1 ) mannitol in the setting of severe traumatic brain injury prior to ICP monitoring be limited to patients with progressive neurological deterioration or signs of transtentorial herniation, and 2) mannitol be dosed in a weight-based fashion at $0.25-1 \mathrm{~g} / \mathrm{kg}$.
The investigation of medical errors is a burgeoning field with limited exposure in the neurosurgical literature. Neurosurgical emergencies are particularly stressful clinical situations for peripheral hospitals that deal with these emergencies infrequently, and therefore these hospitals may be more prone to medical error. Anecdotal experience at our local institution has suggested an incidence of mannitol dosing errors during interhospital transfer for acute neurosurgical conditions. This study examined the frequency of mannitol dosing error in patients being transferred for suspected intracranial emergency. We conducted a retrospective review of patients with intracranial emergency who received mannitol prior to or during helicopter evacuation from the northern half of the province of Alberta, Canada. The study further assessed the appropriateness of mannitol use in this population, specifically the adherence to BTF guidelines.

ABBREVIATIONS BTF = Brain Trauma Foundation; GCS = Glasgow Coma Scale; ICP = intracranial pressure; STARS = Shock Trauma Air Rescue Society. SUBMITTED July 7, 2014. ACCEPTED November 12, 2014.

INCLUDE WHEN CITING Published online June 16, 2015; DOI: 10.3171/2014.11.JNS141596.

DISCLOSURE The authors report no conflict of interest concerning the materials or methods used in this study or the findings specified in this paper. 


\section{Methods}

This project was conducted with the approval of and in accordance with guidelines of the local health ethics research board at the University of Alberta, Edmonton, Alberta, Canada. We electronically searched the Shock Trauma Air Rescue Society (STARS) electronic patient database of all helicopter medical evacuations in Alberta, Canada, limiting our search to patients receiving mannitol infusions prior to or during transfer to a tertiary care center between 2004 and 2012. Specifically, by indexing pretransfer and flight medications, that database search engine allowed us to limit our search to patients who received mannitol. Patient records with an unknown dose, and patients without a documented history of trauma or a neurological deterioration suggestive of elevated ICP, were excluded. Each record was reviewed to extract data on mannitol dose and duration of infusion. Additional extracted data included patient characteristics such as sex, weight, and age; neurological status (pupil size or asymmetry and Glasgow Coma Scale [GCS] score); presumptive diagnosis; and injury mechanism. Because of specified limitations of our use of the electronic database, it was not possible to examine correlation with clinical outcome. Specifically, although the peripheral hospital name and patient identifiers were displayed, ethical constraints prohibited us from recording or analyzing these data.

Assessment of the appropriateness of mannitol use in our patient population was based on the 2007 BTF guidelines on indications for mannitol use prior to ICP monitoring. ${ }^{1}$ Mannitol use was deemed appropriate if patients met any of the following criteria: severe traumatic brain injury or intracranial emergency (defined by a GCS score of 8 or less), a clear neurological deterioration (defined as a documented decline in GCS score of 2 or more points), or pupillary abnormalities indicative of transtentorial herniation or brainstem dysfunction (unilateral or bilateral fixed and/or dilated pupils).

Mannitol dosing error was defined using weight-based dosing guidelines from the BTF of $0.25-1 \mathrm{~g} / \mathrm{kg}$. ${ }^{1}$ Specifically, underdosing was defined as a dose less than 0.25 $\mathrm{g} / \mathrm{kg}$, and overdosing was arbitrarily defined as a dose greater than $1.5 \mathrm{~g} / \mathrm{kg}$ (so as not to include patients in the overdosed group who received an only slightly larger than recommended dose). Nonbolus administration was defined as an infusion time of greater than 60 minutes. To ensure accuracy, mannitol dose and duration of infusion were confirmed by cross-referencing the pretransfer medication list with both the patient event log and the free-text flight $\log$ written by the medical flight crew.

The 2-sample t-test was used to compare means and proportions using SPSS version 20 (IBM).

\section{Results}

This study was conceived following a particularly disconcerting mannitol underdosing error during the evacuation of a 17-year-old female patient with a large acute epidural hematoma that occurred following a downhill skiing accident. This case was not identified or included in our current study because evacuation was carried out via a different (fixed-wing) service that was not indexed by the database we used. The patient presented to a peripheral center following a brief on-hill loss of consciousness, and subsequently her condition deteriorated; a depressed level of consciousness (GCS Score 7) developed, and she exhibited a unilateral fixed, dilated pupil. Upon her arrival at our institution, we realized that the patient had received only $1 \mathrm{~g}$ of mannitol since deterioration of her condition hours earlier (transport was prolonged in this case due to inclement weather). It was this case, which lacked any clear clinical reason for such a small dose of mannitol (e.g., induced hypotension), that prompted our investigation.

During the defined study period, 120 patients were identified as having received mannitol infusion for intracranial emergency initiated at a peripheral hospital and as satisfying the inclusion and exclusion criteria. Patient characteristics (sex, mean age, median GCS score, pupillary examination documentation, number of head $\mathrm{CT}$ scans obtained at the peripheral center, and primary diagnosis) are described in Table 1.

\section{Mannitol Errors}

Overall, there was a $22 \%$ (27 of 120) error rate. Underdosing occurred in $8.3 \%$ of patients (10 of 120). The underdosed group received a mean dose of $0.07 \mathrm{~g} / \mathrm{kg}$. Overdosing occurred in $7.5 \%$ of patients ( 9 of 120). The overdosed group received a mean dose of $1.78 \mathrm{~g} / \mathrm{kg}$. Nonbolus administration occurred in $6.7 \%$ (8 of 120) of patients. The nonbolus administration group had a mean time of infusion of 90 minutes, ranging from 60 to 180 minutes.

\section{Appropriate Indication for Mannitol}

According to the study criteria, $72 \%$ of the patients (86 of 120) had a documented appropriate indication for administration of mannitol. The majority of patients $(69 \%$; 83 of 120) received mannitol because of the severity of their intracranial event as indicated by a GCS score less than 8 . Documented pupillary abnormalities occurred in $25 \%$ of patients ( 30 of 120 ), while neurological deterioration occurred in $11 \%$ (13 of 120). In the $28 \%$ of patients

TABLE 1. Patient characteristics

\begin{tabular}{lc}
\hline \multicolumn{1}{c}{ Characteristic } & Value $^{*}$ \\
\hline Total no. of patients & 120 \\
\hline Sex & $59(49.2)$ \\
\hline Male & $61(50.8)$ \\
\hline Female & 49.0 \\
\hline Mean age in yrs & \\
\hline GCS score & 6 \\
\hline Median & $3-13$ \\
\hline Range & $51(42)$ \\
\hline Pupillary status documented & $5(4)$ \\
\hline Head CT scan prior to transfer & $98(82)$ \\
\hline Primary diagnosis & $13(11)$ \\
\hline Traumatic brain injury & $9(7.5)$ \\
\hline Spontaneous intracranial hemorrhage &
\end{tabular}

\footnotetext{
* Values are presented as the number of patients (\%) unless noted otherwise.
} 
who inappropriately received mannitol (34 of 120), all had a documented GCS score of greater than or equal to 9 (median 11.5). No patients in this group had documented pupillary abnormalities. In fact, $24 \%$ of this group (8 of 34) had a GCS score compatible with mild head injury (GCS Score 13-15). When groups were analyzed separately, the median GCS score of the group who met criteria for mannitol administration prior to ICP monitoring was 5, compared with 11.5 in the group who inappropriately received mannitol. Despite this clinical difference in the severity of head injury between these 2 groups, the mean doses received were $0.82 \mathrm{~g} / \mathrm{kg}$ and $0.83 \mathrm{~g} / \mathrm{kg}$ in the mannitol-appropriate and -inappropriate groups, respectively $(\mathrm{p}>0.05)$. There were no cases of underdosing and only 3 cases of nonbolus administration in the mannitolinappropriate group. When analyzed separately, the mannitol-inappropriate group had a dosing error rate of $18 \%$ (6 of 34), which was not significantly different from the error rate of $24 \%$ (21 of 86 ) in the mannitol-appropriate group $(\mathrm{p}>0.05)$.

\section{Discussion}

Our retrospective review of emergency evacuation to tertiary care centers for intracranial emergency reveals a mannitol dosing error rate of $22 \%$, with $72 \%$ of indexed patients meeting our clinical criteria for appropriate mannitol use. This error rate is substantially higher than the reported prevalence of medication errors in the general inpatient hospital care setting (approximately 1.5\%).,7 Medication error in the intensive care setting is higher, occurring at a median rate of 106 medication errors per 1000 patient-days. ${ }^{5,6,9}$

The effect of mannitol on ICP is dose dependent, with higher doses $(1 \mathrm{~g} / \mathrm{kg})$ providing more sustained control of ICP. ${ }^{4,8}$ Continuous infusions of mannitol take longer to lower ICP and have not been found to have any benefit over bolus administration. ${ }^{4}$ Therefore, the most potentially harmful and also most common type of error in this study is underdosing, which occurred in $8 \%$ of cases in this study. Particularly worrisome is the finding that the underdosed group received a mean dose of only $0.07 \mathrm{~g} / \mathrm{kg}$, which is significantly lower than the minimal dose recommended by the BTF guidelines of $0.25 \mathrm{~g} / \mathrm{kg}$. Similarly, the $7 \%$ nonbolus administration rate, which occurred in a completely distinct set of patients, would also fail to effect a rapid decrease in ICP.

Analysis of the potential cause of the errors described in this study was limited by the detail that the reviewed database contained. For example, we were not able to review the written physician orders but rather only the documentation of the orders being given in the form of the flight medication administration record and preflight medication record. Given these limitations, analysis of the underdosing errors revealed a potential source of error, that is, unit confusion, with clinicians ordering 100 $\mathrm{mg}$ of mannitol instead of the correct 100-g dose. Another possible cause of underdosing is the concern of inducing hypotension in the setting of trauma. Examination of the medication administration record and the patient care notes did not identify any cases in which mannitol was stopped for any reason (e.g., hypotension), and we did not find instances of mannitol given in multiple smaller boluses. Another potential source of error is conversion of volume to mass or vice versa. In tertiary centers, mannitol is most commonly formulated in a $20 \%$ solution; however, in some hospitals where it is used infrequently it may require reconstitution from solid form. Mass-based orders will typically require conversion into a volume by the prescriber or the individual administering the medication, which introduces another potential source for errors to occur. Finally, we speculate that failing to specify a time over which the bolus is to be given (i.e., "rapid bolus" or "over 5 minutes") may contribute to the chance of a nonbolus administration error occurring. Due to limits placed on our usage of this evacuation database-namely, that we were not able to record patient-identifying information or peripheral hospital name-we were not able to assess the clinical impact of mannitol dosing error or the relative importance to clinical outcome of duration of transfer. Similarly, due to limitations in the level of detail available in the database, we were not able to directly assess any delay in transport attributable to mannitol administration (e.g., Foley catheter placement or mannitol reconstitution); however, there were no instances of hypotension recorded following administration of mannitol.

Overdosing was found in $7.5 \%$ of transferred patients, who as a group received an average dose of $1.78 \mathrm{~g} / \mathrm{kg}$. These errors are likely to be of less clinical significance, especially given work by Cruz and colleagues demonstrating more frequent improvement of pupillary abnormalities as well as improved survival (6-month mortality $39.1 \%$ vs $66.7 \%$, respectively) with high-dose mannitol $(\sim 1.4 \mathrm{~g} / \mathrm{kg})$ versus the standard dose $(\sim 0.7 \mathrm{~g} / \mathrm{kg})$ for severe diffuse brain swelling. ${ }^{2}$ These authors advocated that the mannitol bolus be routinely followed by a rapid bolus of intravenous normal saline to avoid acute hypovolemia and cerebral hypoperfusion. Certainly, in our review, there were no cases of induced hypotension in patients who received high-dose mannitol therapy.

Our secondary objective was to assess the appropriateness of mannitol use within our study group. Although consensus regarding the optimal dosage of mannitol for treatment of ICP is lacking, widely used guidelines do exist that outline indications and effective dose. The 2007 BTF guidelines based on best available evidence suggest weight-based dosing of mannitol at $0.25-1 \mathrm{~g} / \mathrm{kg}$ should be limited to use for intracranial emergency prior to ICP monitoring in patients with progressive neurological deterioration or signs of transtentorial herniation. ${ }^{1}$ Using these guidelines, we found that $72 \%$ of patients had a clear indication for mannitol, with $11 \%$ having a documented neurological deterioration, $30 \%$ having pupillary abnormalities suggestive of herniation, and $69 \%$ meeting criteria for severe brain injury. Patients who received mannitol inappropriately had on average a higher level of consciousness (as indicated by GCS scores), but the dosing error rate in these patients was similar to that in the mannitol-appropriate group (18\% vs $24 \%$ ) and did not account for any of the underdosing errors. Three patients in the mannitolinappropriate group received nonbolus administration.

Potential limitations of our study included the reliance 
of our error analysis on the information contained in the flight case $\log$ created by the medical team involved in evacuation. Therefore, we were not able to directly review the physicians' orders or to compare the orders to the medication administration record to check for inconsistencies. Second, our study was not able to assess the clinical impact of the mannitol errors or to determine the cause of such errors because we were not permitted to use patient identifiers from the non-hospital based STARS electronic patient database.

Finally, our findings suggest that strategies such as a preflight checklist of mannitol dose in the setting of evacuation for intracranial emergency may mitigate potentially harmful underdosing and nonbolus administration errors. In collaboration with STARS staff we plan to introduce such a checklist along with medication error awareness sessions for a period of 2 years, after which we will reevaluate rates of mannitol error during evacuations for intracranial emergencies. Gathering data prospectively following implementation of this program will allow us to examine the clinical impact of mannitol errors as well as duration of transport stratified by severity of injury.

\section{Conclusions}

Mannitol administration during interhospital evacuation for intracranial emergency is prone to dosing error. Clinicians should specify the volume, concentration, and time of administration when ordering mannitol.

\section{References}

1. Bratton SL, Chestnut RM, Ghajar J, McConnell Hammond FF, Harris OA, Hartl R, et al: Guidelines for the management of severe traumatic brain injury. II. Hyperosmolar therapy. J Neurotrauma 24 (Suppl 1):S14-S20, 2007

2. Cruz J, Minoja G, Okuchi K, Facco E: Successful use of the new high-dose mannitol treatment in patients with Glasgow Coma Scale scores of 3 and bilateral abnormal pupillary widening: a randomized trial. J Neurosurg 100:376-383, 2004

3. Dean B, Schachter M, Vincent C, Barber N: Causes of pre- scribing errors in hospital inpatients: a prospective study. Lancet 359:1373-1378, 2002

4. Fink ME: Osmotherapy for intracranial hypertension: mannitol versus hypertonic saline. Continuum (Minneap Minn) 18:640-654, 2012

5. Kane-Gill S, Weber RJ: Principles and practices of medication safety in the ICU. Crit Care Clin 22:273-290, 2006

6. Kane-Gill SL, Jacobi J, Rothschild JM: Adverse drug events in intensive care units: risk factors, impact, and the role of team care. Crit Care Med 38 (6 Suppl):S83-S89, 2010

7. Nichols P, Copeland TS, Craib IA, Hopkins P, Bruce DG: Learning from error: identifying contributory causes of medication errors in an Australian hospital. Med J Aust 188:276-279, 2008

8. Sorani MD, Manley GT: Dose-response relationship of mannitol and intracranial pressure: a metaanalysis. J Neurosurg 108:80-87, 2008

9. Valentin A, Capuzzo M, Guidet B, Moreno RP, Dolanski L, Bauer P, et al: Patient safety in intensive care: results from the multinational Sentinel Events Evaluation (SEE) study. Intensive Care Med 32:1591-1598, 2006

\section{Author Contributions}

Conception and design: all authors. Acquisition of data: Elliott. Analysis and interpretation of data: all authors. Drafting the article: Elliott. Critically revising the article: all authors. Reviewed submitted version of manuscript: all authors. Statistical analysis: O'Kelly, Elliott. Study supervision: O'Kelly.

\section{Supplemental Information}

Previous Presentation

Portions of this work were presented in abstract form as proceedings at the 15th WFNS World Congress of Neurosurgery, Seoul, South Korea, September 8-13, 2013, as well as the 48th Annual Congress of the Canadian Neurological Sciences Federation, Montreal, Quebec, Canada, June 12-14 2013.

\section{Correspondence}

Cian J. O'Kelly, Division of Neurosurgery, 2D2.01, Walter Mackenzie Health Centre, 8840112 St. NW, Edmonton, AB T6G 2B7, Canada. email: cian.okelly@albertahealthservices.ca. 